\title{
Kynurenine derivative 3-HAA is an agonist ligand for transcription factor YY1
}

\author{
Zhaopeng Shi ${ }^{1 \dagger}$, Guifang Gan ${ }^{1 \dagger}$, Xiang Xu' ${ }^{1}$, Jieying Zhang ${ }^{1}$, Yuan Yuan ${ }^{1}$, Bo Bi ${ }^{2}$, Xianfu Gao ${ }^{3}$, Pengfei Xu ${ }^{4}$, \\ Wenbin Zeng ${ }^{4}$, Jixi Li ${ }^{5}$, Youqiong Ye ${ }^{1}$, Aiwu Zhou ${ }^{1}$, Naixia Zhang ${ }^{6}$, Wen Liư ${ }^{7^{*}}$, Shuhai Lin ${ }^{8^{*}}$ and Jun Mi ${ }^{1,2^{*}}$ (D)
}

\begin{abstract}
The 3-hydroxyanthranilic acid (3-HAA), a derivative of kynurenine, was reported to suppress tumor growth. However, the function of 3-HAA largely remains unclear. Here, we report that 3-hydroxyanthranilic acid (3-HAA) is lower in tumor cells, while adding exogenous 3-HAA induces apoptosis in hepatocellular carcinoma by binding YY1. This 3-HAA binding of YY1 leads to phosphorylation of YY1 at the Thr 398 by PKC, concomitantly enhances YY1 chromatin binding activity to increase expression of target genes. These findings demonstrate that 3-HAA is a ligand of YY1, suggesting it is a promising therapeutic candidate for HCC.
\end{abstract}

Keywords: 3-Hydroxyanthronic acid (3-HAA), Kynurenine, YY1, DUSP6, Hepatocellular carcinoma (HCC), Tryptophan metabolism

\section{Highlights}

1. 3-HAA induces apoptosis of HCC cells by binding YY1

2. 3-HAA recruits $\mathrm{PKC \zeta}$ to phosphorylate T398 of YY1

Tryptophan metabolism is enhanced in various tumors by upregulating the indoleamine 2,3-dioxygenase $1 / 2$ (IDO1/2) or tryptophan 2,3-dioxygenase (TDO2) [1-3]. The 3-hydroxyanthranilic acid (3-HAA), a derivative of kynurenine, was reported to suppress tumor growth [4]. However, the function of 3-HAA largely remains unclear.

\footnotetext{
*Correspondence: w2liu@xmu.edu.cn; shuhai@xmu.edu.cn; jmei@sjtu.edu.cn ${ }^{\dagger}$ Zhaopeng Shi and Guifang Gan have contributed equally to this work ${ }^{1}$ Basic Medical Institute, Key Laboratory of Cell Differentiation and Apoptosis of the Chinese Ministry of Education, Hongqiao International Institute of Medicine, Tongren Hospital, Shanghai Jiao Tong University School of Medicine, Shanghai, China

${ }^{7}$ School of Pharmaceutical Sciences, State Key Laboratory of Cellular Stress Biology, Xiamen University, Xiamen, China

${ }^{8}$ School of Life Sciences, State Key Laboratory of Cellular Stress Biology, Xiamen University, Xiamen, China

Full list of author information is available at the end of the article
}

\section{3-HAA induces apoptosis by binding with transcription factor YY1}

Utilizing liquid chromatography-tandem mass spectrometry (LC-MS/MS), we first found that the concentration of 3-HAA decreased in 37 cases of HCC $(p<0.01)$ compared to the matched paratumor tissues (Fig. 1A). The gene ontology $(\mathrm{GO})$ analysis revealed that the apoptosis pathway was highly activated in 3-HAA-treated HCC cells (Additional file 1: Fig. S1A), which was confirmed by the TUNEL assay that apoptosis was increased in a dosedependent manner in 3-HAA-treated SMMC7721 cells and HCC xenografts (Fig. 1B, C). Levels of cleaved caspase 3 and cleaved PARP were increased in a dose- and time-dependent manner in the SMMC7721 and HepG2 cells (Additional file 1: Fig. S1B). Consequently, 3-HAA treatment led to suppression of HCC xenografts growth (Fig. 1D).

The gene expression profiles of SMMC7721 or HepG2 cells were further analyzed, and the top 21 upregulated genes at all three-time points $(1,8$, or $24 \mathrm{~h})$ after the start of 3-HAA treatment were selected (Additional file 1: Fig. $\mathrm{S} 1 \mathrm{C})$. The 38 common transcription factors were first selected from those proteins that potentially bind to the 
promoter region $(-5000$ to +1$)$ of the top 4 genes [5] (Fig. 1E and Additional file 1: Fig. S1D). Through tandem mass-tagged quantitative proteomics analysis, the 91 proteins are increasingly bound to chromatin from the $1^{\text {st }}$ hour to the $8^{\text {th }}$-hour post-3-HAA treatment (Additional file 1: Fig. S1E), and YY1 was the only protein overlapped with the predicted transcription factors that potentially bound to the promoter region of the top 4 genes (Fig. 1F). The function assay showed that YY1 knockdown abolished 3-HAA-induced upregulation of target genes in SMMC7721 cells (Fig. 1G), and 3-HAA-induced apoptosis was reduced in SMMC7721 cells depleted of YY1 (Fig. 1H, Additional file 1: Figs. S1F, and S1G). Consequently, 3-HAA reduced tumor numbers and prolonged mice survival in mice with transposon-induced HCCs, whereas the same dose of 3-HAA had no remarkable effect on tumor numbers and mice survival once YY1 depleted.

The ChIP-sequencing analysis demonstrated that 3-HAA induced the union peak formation of YY1 on the promoter region of the top 4 genes (Fig. 1J \& Additional file 1: Fig. S1I). 3-HAA increased YY1 binding to the promoter sequence of DUSP6 in a dose-dependent manner, as evidenced by an in vitro electrophoretic mobility shift assay (Fig. 1K). Furthermore, nuclear magnetic resonance was performed to determine whether 3-HAA directly binds YY1. Dose-dependent signal attenuation was observed in the T1r NMR spectrum, suggesting that YY1 directly interacts with 3-HAA (Fig. 1L).

\section{PKCZ phosphorylates YY1 at Thr 398 in response to 3-HAA}

Thus, the YY1 phosphorylation was further analyzed to determine whether 3-HAA induced YY1 phosphorylation. The immunoblotting following phosphorylation protein enrichment showed that 3-HAA increased YY1 phosphorylation, and mass spectrometry analysis revealed that the T398 of YY1 was phosphorylated in 3-HAA-treated HCC cells (Fig. 1A). The T398A mutation diminished the 3-HAA-induced YY1 T398 phosphorylation (Fig. 2B). The function analysis displayed that the T398A mutation of YY1 suppressed 3-HAA-upregulated DUSP6 expression and reduced the level of cleaved Caspase 3/cleaved PARP, whereas the mimic phosphorylation of T398E mutation promoted DUSP6 expression even without 3-HAA treatment (Fig. 2C). The TUNEL assay and the flow cytometry analysis demonstrated that the T398A mutation of YY1 suppressed 3-HAA-induced apoptosis (Fig. 2D \& Additional file 1: Fig. S2A), suggesting T398 phosphorylation of YY1 is critical for 3-HAAinduced apoptosis.

Moreover, the kinase screening assay was performed on the FAQSTNLK peptide of YY1. Proteomic analysis by mass spectrometry following YY1 immunoprecipitation showed that 3-HAA increased the association of YY1 with PKCद, which were cosistent with the kinase prediction $[6,7]$ and further confirmed by immunoblotting, suggesting that 3-HAA recruits $\mathrm{PKC} \zeta$ to phosphorylate YY1 (Additional file 1: Figs. S2B, Fig. 2E, F). The kinase $\mathrm{PKC \zeta}$ significantly increased the peptide phosphorylation, reflected by the autoradiogram on the dot blot (Fig. 2G). Also, only the PKC decreased YY1 T398 phosphorylation (Fig. 2H), suggesting that $\mathrm{PKC \zeta}$ is the kinase for T398 phosphorylation of YY1 induced by 3-HAA.

Moreover, the T398E mutation but not T398A mutation of YY1 increase the YY1 binding on the DUSP6 promoter, no matter with or without 3-HAA, and the PKCל inhibitor markedly decreased the YY1 binding on DUSP6 promoter in the SMMC7721 cells depleted of endogenous YY1 and expressing exogenous wild type YY1, but not in the cells expressing T398A/T398E mutant YY1 (Fig. 2I). Also, 3-HAA had no effect on tumor growth

\footnotetext{
(See figure on next page.)

Fig. 13 -HAA induces apoptosis by binding with transcription factor YY1. A Quantitative analysis of tryptophan metabolites by LC-MS/MS and GC-MS in HCC and the corresponding paratumor tissues. ${ }^{* *}: P<0.01$. B Apoptosis analysis by TUNEL assay in SMMC7721 cells treated for $24 \mathrm{~h}$ with 3-HAA at the indicated dose. ${ }^{* *}: P<0.01$. C Apoptosis analysis by TUNEL assay and flow cytometry in SMMC7721 xenografts with and without 3-HAA treatment. The dose of 3-HAA was $100 \mathrm{mg} / \mathrm{kg}$ day. Mice were treated for seven days and sacrificed on day $10 . *: P<0.05$. D The growth curve and representative photo of PCX xenografts. $\mathbf{E}$ The common proteins between 3-HAA-increased chromatin-binding proteins and predicted transcription factors binding to the top 4 genes' promoter region. F The common proteins between 3-HAA-increased chromatin-binding proteins and predicted transcription factors binding to the promoter region of the top 4 genes. G YY1 knockdown abolished 3-HAA-induced DUSP6 expression. SMMC7721 cells were treated with $100 \mu \mathrm{M}$ of 3-HAA for the indicated time. HThe effect of YY1 knockdown on 3-HAA-induced HCC apoptosis, analyzed by flow cytometry. The concentration of 3-HAA was $100 \mu \mathrm{M}$. The **: $P<0.01$. IYY1 depletion attenuated 3-HAA suppression of HCC tumor formation and shorten the survival of mice bearing transposon HCCs. The transposon genetic HCC mouse model was established as described in the section of Methods and Materials $(n=6)$. J The ChIP-sequencing analysis of YY1 on the DUSP6 and UGFBP1 genes. The HCC cells were treated with the indicated dose of 3-HAA prior to ChIP-sequencing. K 3-HAA binding to YY1 was determined by electrophoretic mobility shift assay (EMSA). The synthesized oligonucleotide was labeled at the $5^{\prime}$ terminus with FAM, and YY1 was purified using a Hitrap heparin column. L NMR measurement of direct binding between 3-HAA and YY1.T1r NMR spectra for PBS (red) alone or in the presence of YY1 at $5 \mu M$ (green), or $8 \mu M$ (blue). The CPMG spectrum for $6 \mathrm{~b}$ was recorded in the presence of $5 \mathrm{mM} \mathrm{YY} 1$
} 


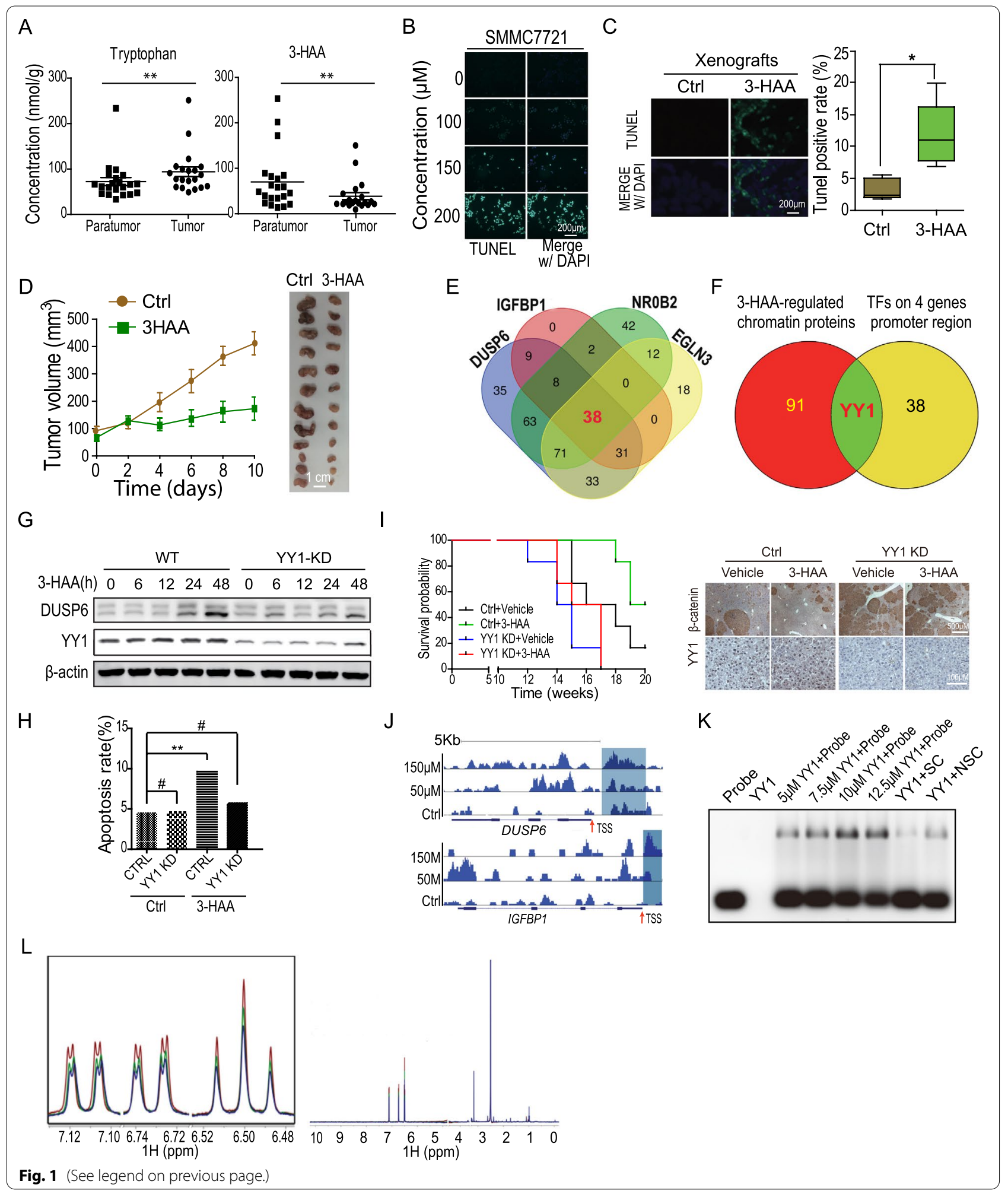



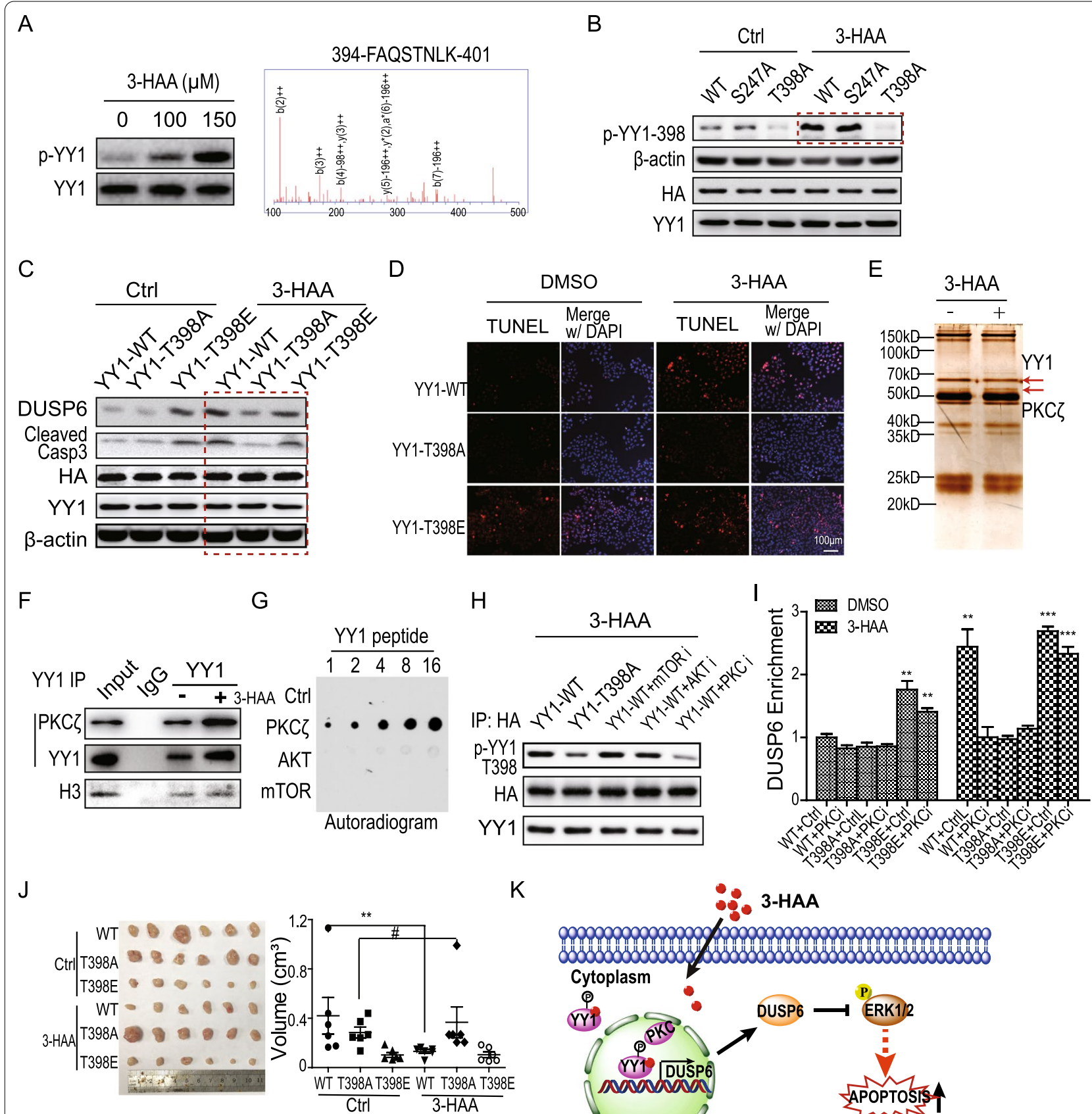

K

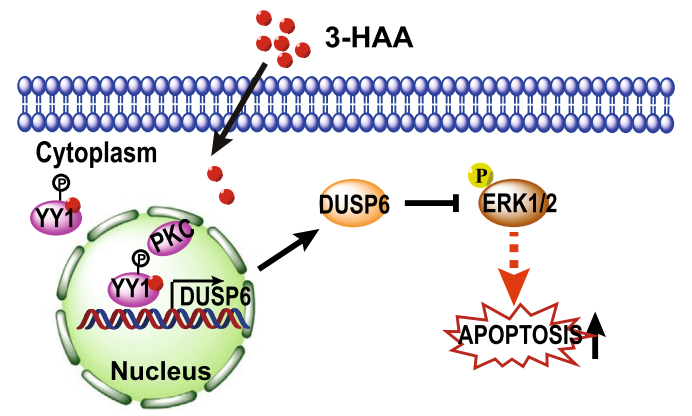

Fig. 2 (See legend on next page.)

in mice with HCC xenograft depleting endogenous YY1 and expressing T398A mutant YY1. Whereas the same dose of 3-HAA significantly decreased xenograft growth expressing wild type YY1 (Fig. 2J). The clinical data that the PKC expression level was closely correlated with the overall survival of the grade I HCC patients (Additional file 1: Fig. S2C) further supported these findings.
In brief, our results have determined that 3-HAA is an active metabolite regulating tumor cell fate by binding to and activating the transcription factor YY1 (Fig. 2K). The T398 phosphorylation of YY1 promots YY1 binding to its target sequence. Exogenous 3-HAA induces tumor cell apoptosis and inhibits HCC growth, suggesting its potential use in HCC therapy. 


\section{(See figure on previous page.)}

Fig. 2 PKC phosphorylates YY1 at Thr 398 in response to 3-HAA. A The YY1 phosphorylation was analyzed by immunoblotting and mass spectrometry $2 \mathrm{~h}$ after 3-HAA treatment. The YY1 was blotted on the enriched phospho-proteins from SMMC7721 cells. The YY1 modification was analyzed by the mass-spectrometry following YY1 immunoprecipitation. B The T398A but not S247A mutation abolished 3-HAA-induced YY1 phosphorylation. The YY1 was conjugated with HA tag. The YY1 phosphorylation was detected by the T398 phospho-specific antibody. C The mutation of T398E in YY1 promoted DUSP6 expression and apoptosis, whereas the T398A mutation suppressed DUSP6 upregulation. The YY1 was fused with HA tag. D The YY1 mutation of T398A reduces the 3-HAA-induced apoptosis, analyzed by the TUNEL assay. E 3-HAA increased PKC binding to YY1, analyzed by co-immunoprecipitation. F 3-HAA increased PKCZ binding to YY1, analyzed by immunoblots following immunoprecipitation. G The kinase screening for T398 phosphorylation of YY1. The kinase candidates were predicted by online tools NetPhos 3.1 (www.cbs.dtu.dk/services/NetPhos) [8] and GPS 5.0 (gps.biocuckoo.cn) [9]. H The effect of kinase inhibitors on 3-HAA-induced YY1 phosphorylation. The YY1 phosphorylation was detected by the T398 phospho-specific antibody. The concentration of 3-HAA was $100 \mu$ M. AKT inhibitor, MK2206

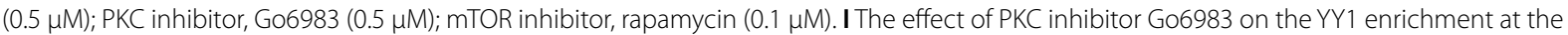
DUSP6 promoter in SMMC-7721 cells. **: $P<0.01$, ***: $P<0.001$. J The T398A mutation of YY1 abolished the 3-HAA-inhibited HCC xenografts growth $(\mathrm{n}=6){ }^{* *}: P<0.01 . \mathbf{F}$ and $\mathbf{G}$ The apoptosis analysis in xenografts by TUNEL assay and the flow cytometry. The histogram presents as mean $\pm S D(*$ : $P<0.05 ;{ }^{*}: P<0.01$.). Note: The dose of 3-HAA used in $\mathbf{A}$ was $100 \mu \mathrm{M}$. $\mathbf{K}$ The proposed 3-HAA binding model with $\mathrm{YY} 1$

\section{Supplementary Information}

The online version contains supplementary material available at https://doi. org/10.1186/s13045-021-01165-4.

Additional file 1. Supplemental Figures \& the Methods and Material.

\section{Acknowledgements}

We thank Profs. Lei Shen (Shanghai Jiao Tong University School of Medicine) and Qunying Lei (Fudan University School of Medicine) for useful discussions.

\section{Authors' contributions}

Z.S., G.G., S.L. and X.X. performed most of experiments; Y.Y., J.Z. and B.B. performed some of animal experiments; X.G. performed metabolites analysis, P.X. and W.Z. synthesize the 3-HAA derivatives; N.Z. and A.Z. performed the 3-HAA and $Y Y 1$ binding experiments; W.L. performed ChIP-Seq analysis and kinase screening; J.M. initiated the project, led the project team, designed experiments, analyzed results, and wrote the paper with input from all authors. All authors read and approved the final manuscript.

\section{Funding}

This study was supported by grants from the Ministry of Science and Technology of the People's Republic of China (2018YFC1313205), the Shanghai Committee of Science and Technology (11DZ2260200) (20JC1410100) and the National Natural Science Foundation of China (81572300) (81872342) to Dr. Mi and (91957120) to Dr. Lin.

\section{Declarations}

\section{Ethical approval and consent to participate}

This study received animal ethics board approval at the Shanghai Jiao Tong University School of Medicine.

\section{Consent for publication}

Not applicable.

\section{Competing interests}

The authors declare that they have no competing interests.

\section{Author details}

${ }^{1}$ Basic Medical Institute, Key Laboratory of Cell Differentiation and Apoptosis of the Chinese Ministry of Education, Hongqiao International Institute of Medicine, Tongren Hospital, Shanghai Jiao Tong University School of Medicine, Shanghai, China. ${ }^{2}$ Department of Nuclear Medicine, Rui Jin Hospital, Shanghai Jiao Tong University School of Medicine, Shanghai, China. ${ }^{3}$ Shanghai Profleader Biotech Co., Ltd, Shanghai, China. ${ }^{4}$ Xiangya School of Pharmaceutical Sciences, Central South University, Changsha, China. ${ }^{5}$ School of Life Science, Fudan University, Shanghai, China. ${ }^{6}$ CAS Key Laboratory of Receptor Research,
Department of Analytical Chemistry, Shanghai Institute of Materia Medica, Chinese Academy of Sciences, Shanghai, China. ${ }^{7}$ School of Pharmaceutical Sciences, State Key Laboratory of Cellular Stress Biology, Xiamen University, Xiamen, China. ${ }^{8}$ School of Life Sciences, State Key Laboratory of Cellular Stress Biology, Xiamen University, Xiamen, China.

Received: 4 August 2021 Accepted: 7 September 2021

Published online: 25 September 2021

\section{References}

1. Terai M, Londin E, Rochani A, Link E, Lam B, Kaushal G, Bhushan A, Orloff $M$, Sato T. Expression of tryptophan 2,3-dioxygenase in metastatic uveal melanoma. Cancers (Basel). 2020;12:405.

2. Li S, Weng J, Song F, Li L, Xiao C, Yang W, Xu J. Circular RNA circZNF566 promotes hepatocellular carcinoma progression by sponging miR4738-3p and regulating TDO2 expression. Cell Death Dis. 2020;11:452.

3. Sadik A, Somarribas Patterson LF, Ozturk S, Mohapatra SR, Panitz V, Secker PF, Pfander P, Loth S, Salem H, Prentzell MT, et al. IL4I1 is a metabolic immune checkpoint that activates the AHR and Promotes Tumor progression. Cell. 2020;182:1252-70.

4. Gan G, Shi Z, Shangguan C, Zhang J, Yuan Y, Chen L, Liu W, Li B, Meng S, Xiong W, Mi J. The kynurenine derivative 3-HAA sensitizes hepatocellular carcinoma to sorafenib by upregulating phosphatases. Theranostics. 2021;11:6006-18.

5. Yevshin I, Sharipov R, Kolmykov S, Kondrakhin Y, Kolpakov F. GTRD: a database on gene transcription regulation-2019 update. Nucleic Acids Res. 2019;47:D100-5.

6. Xue Y, Liu Z, Cao J, Ma Q, Gao X, Wang Q, Jin C, Zhou Y, Wen L, Ren J. GPS 2.1: enhanced prediction of kinase-specific phosphorylation sites with an algorithm of motif length selection. Protein Eng Des Sel. 2011;24:255-60.

7. Mok J, Kim PM, Lam HY, Piccirillo S, Zhou X, Jeschke GR, Sheridan DL, Parker SA, Desai V, Jwa M, et al. Deciphering protein kinase specificity through large-scale analysis of yeast phosphorylation site motifs. Sci Signal. 2010;3:ra12.

8. Blom N, Sicheritz-Ponten T, Gupta R, Gammeltoft S, Brunak S. Prediction of post-translational glycosylation and phosphorylation of proteins from the amino acid sequence. Proteomics. 2004;4:1633-49.

9. Song C, Ye M, Liu Z, Cheng H, Jiang X, Han G, Songyang Z, Tan Y, Wang H, Ren J, et al. Systematic analysis of protein phosphorylation networks from phosphoproteomic data. Mol Cell Proteom. 2012;11:1070-83.

\section{Publisher's Note}

Springer Nature remains neutral with regard to jurisdictional claims in published maps and institutional affiliations. 